\title{
MARSHALL-PEIERLS SIGN RULE IN FRUSTRATED HEISENBERG CHAINS
}

\author{
A. VoIgt AND J. Richter \\ Institut für Theoretische Physik, Otto-von-Guericke-Universität Magdeburg \\ Postfach 4120, 39106 Magdeburg, Germany
}

\begin{abstract}
We consider the frustrated antiferromagnetic $s=1$ Heisenberg quantum spin chain with regard to the Marshall-Peierls sign rule. Using exact diagonalization data we investigate the breakdown of the Marshall-Peierls sign rule in dependence on frustration and compare our findings with data for $s=1 / 2$. We calculate a critical value of frustration $J_{2}^{\text {crit }}$, where the Marshall-Peierls sign rule is violated. The extrapolation of this value to the infinite chain limit holds $J_{2}^{\text {crit }} \approx 0.016$, lower than in the case of $s=1 / 2$ $\left(J_{2}^{\text {crit }} \approx 0.027\right)$. This points to a stronger influence of frustration in the case of $s=1$. Nevertheless, the calculation of the weight of the Ising-states violating the Marshall-Peierls sign rule shows that the latter holds approximately even for a quite large frustration and may be used for numerical techniques.

PACS numbers: $75.10 . \mathrm{Jm}, 75.40 . \mathrm{Mg}, 75.50$.Ee
\end{abstract}

\section{Introduction}

The Marshall-Peierls sign rule (MPSR) determines the sign of the Ising-basis-states building the ground-state wave function of a Heisenberg Hamiltonian [1] and has been proven exactly for bipartite lattices and arbitrary site spins by Lieb, Schultz and Mattis [2]. As pointed out in several papers the knowledge of the sign is of great importance in different numerical methods, e.g. for the construction of variational wave functions [3], in quantum Monte-Carlo methods (which suffer from the sign problem in frustrated systems [4]) and also in the density matrix renormalization group method, where the application of the MPSR has the substantially improved method in a frustrated spin system [5].

The MPSR has been analyzed so far for systems with $s=1 / 2$. The authors of Ref. [6] studied the frustrated chain and found for the ground state a critical value for the breakdown of the MPSR for the infinite chain limit using exact diagonalization data. For the $J_{1}-J_{2}$ model on the square lattice the violation of the MPSR was considered as an indication for the breakdown of long range order $[7,8]$. In the recent paper [9] we extended these investigations to higher subspaces of $S^{z}$. For linear chains we have shown that for the lowest eigenstates in every subspace $S^{z}$ there is a finite region of frustration where the MPSR holds. 
In this paper we want to analyze the frustrated spin chain with $s=1$. This spin system has attracted a lot of attention, because of the well-known Haldane conjecture [10]. The unfrustrated $s=1$ spin chain shows a spin gap and exponential decaying correlations whereas the $s=1 / 2$ spin chain has no gap and a power-law correlation decay. Since both systems are qualitatively different one might expect also a different influence of frustration on the MPSR.

\section{The model and the Marshall-Peierls sign rule}

In the following we study the MPSR for the frustrated antiferromagnetic $s=1$ Heisenberg quantum spin chain

$$
\widehat{H}=J_{1} \sum_{\langle\mathrm{nn}\rangle} s_{i} s_{j}+J_{2} \sum_{\langle\mathrm{nnn}\rangle} s_{i} s_{j},
$$

where $\langle\mathrm{nn}\rangle$ and $\langle\mathrm{nnn}\rangle$ denote nearest-neighbor and next-nearest-neighbor bonds on the linear chain. We set $J_{1}=1$ for the rest of the paper. For this model the MPSR can be exactly proved only for $J_{2} \leq 0$.

The Marshall-Peierls sign rule can be described as follows: In the unfrustrated limit of $J_{2}=0$, the lowest eigenstate of the Hamiltonian (1) in each subspace of fixed eigenvalue $M$ of the spin operator $S_{\text {total }}^{z}$ reads

$$
\Psi_{M}=\sum_{m} c_{m}^{(M)}|m\rangle, \quad c_{m}^{(M)}>0 .
$$

Here the Ising-states $|m\rangle$ are defined by

$$
|m\rangle \equiv(-1)^{S_{A}-M_{A}}\left|m_{1}\right\rangle \otimes\left|m_{2}\right\rangle \otimes \cdots \otimes\left|m_{N}\right\rangle
$$

where $\left|m_{i}\right\rangle, i=1, \cdots, N$, are the eigenstates of the site spin operator $S_{i}^{z}$ $\left(-s_{i} \leq m_{i} \leq s_{i}\right), S_{A}=\sum_{i \in A} s_{i}, M_{A(B)}=\sum_{i \in A(B)} m_{i}, M=M_{A}+M_{B}$. The lattice consists of twoequivalent sublattices $A$ and $B . s_{i} \equiv s, i=1, \cdots, N$, are the site spins. The summations in Eq. (2) are restricted by the condition $\sum_{i=1}^{N} m_{i}=M$. The wave function (2) is not only an eigenstate of the unfrustrated Hamiltonian $\left(J_{2}=0\right)$ and $S_{\text {total }}^{z}$ but also of the square of the total spin $S_{\text {total }}^{2}$ with quantum number $S=|M|$. Because $c_{m}^{(M)}>0$ is valid for each $m$ from the basis set (3) it is impossible to build up other orthonormal states without using negative amplitudes $c_{m}^{(M)}$. Hence the ground-state wave function $\Psi_{M}$ is nondegenerated. As it comes out, the MPSR is still fulfilled not only for the ground state but also for every lowest eigenstate in the subspace $M$ in the unfrustrated case. We emphasize that for $J_{2}>0$ no proof for the above statements can be given and that a frustrating $J_{2}>0$ can destroy the MPSR.

\section{Results}

We have calculated the ground state of the model (1) for $N=8, \ldots, 14$ varying the frustration parameter $J_{2}$ by exact diagonalization. While analyzing the ground-state wave function according to the MPSR we found for every system a critical value of frustration $J_{2}^{\text {crit }}$, where the MPSR starts to be violated. We apply the scaling law proposed by Zeng and Parkinson [6] and extrapolate our data as a function of $1 / N^{2}$. We found a value for the infinite chain limit: 


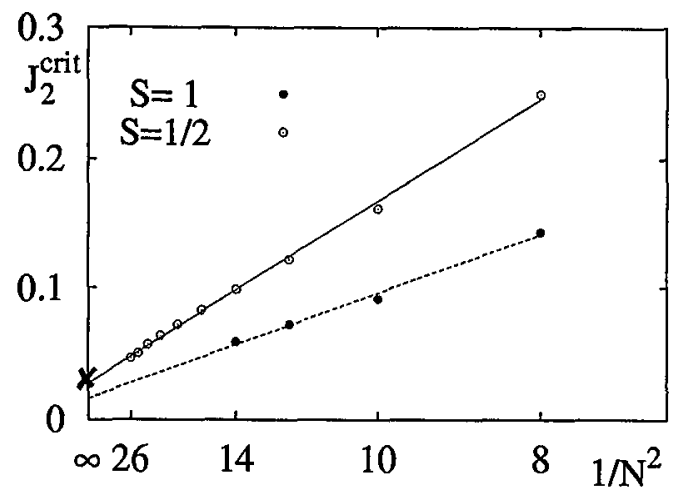

Fig. 1. The critical value of frustration $J_{2}^{\text {crit }}$, where the MPSR starts to be violated as a function of the system size $N$. The cross denotes the value of Zeng and Parkinson [6].

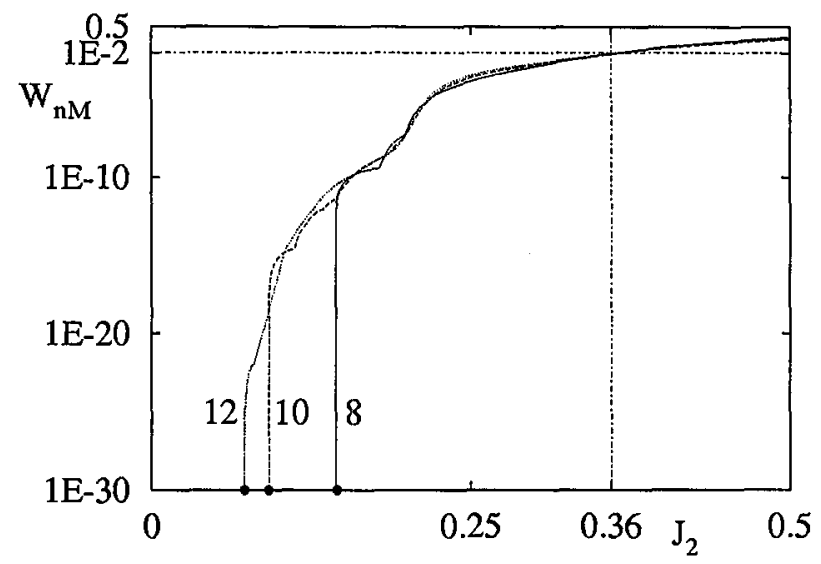

Fig. 2. The weight of non-Marshall-states of the ground-state wave function $W_{n M}$ as a function of frustration $J_{2}$ for systems with $N=8,10,12$.

$J_{2}^{\text {crit }}(\infty)=0.016 \pm 0.003$. In Fig. 1 we compare these data with the values for the $s=1 / 2$ systems $(N=8, \ldots, 26)$, where the extrapolation yields $J_{2}^{\text {crit }}(\infty)=$ $0.027 \pm 0.003$. It is also interesting to note that this value is slightly lower than the value of 0.032 found by Zeng and Parkinson [6] using data for $N=8, \ldots, 20$ only.

We argue that in the case of $s=1$ the chain is more sensitive to frustration and therefore the MPSR is violated for smaller values of $J_{2}$. Nevertheless, in numerical methods the MPSR can be used at least approximately for much larger values of frustration. This can be justified by the examination the ground-state wave function according to the Ising-basis-states which violates the MPSR. We call these states non-Marshall-states and denote their weight by $W_{n M}$. In Fig. 2 we show $W_{n M}$ as a function of frustration $J_{2}$.

As can be seen in Fig. 2, the weight of the non-Marshall-states remains smaller than $1 \%(1 \mathrm{E}-2)$ until $J_{2} \approx 0.36$. This result seems to be more or less size independent because all three lines for the systems with $N=8,10$, and 12 cross at this point. The points at the bottom line denote the first violation of the MPSR 
in a given system and coincide with the points given in Fig. 1. The examination of $W_{n M}$ indicates that for a quite large frustration the predominant part of the ground-state wave function fulfills the MPSR. Therefore, the MPSR can be used in numerical methods even if it does not hold strictly.

\section{Conclusions}

We have shown that in the frustrated antiferromagnetic $s=1$ Heisenberg quantum spin chain the Marshall-Peierls sign rule is violated by frustration. By extrapolation to the infinite chain limit we found a critical value of frustration $J_{2}^{\text {crit }} \approx 0.016 \pm 0.003$ below which the MPSR still holds exactly. By calculating the weight of the Ising-basis-states of the ground-state wave function which do not fulfill the MPSR we conclude that the MPSR can be used in numerical methods at least approximately until a large frustration of $J_{2} \approx 0.36$.

\section{Acknowledgments}

We would like to thank Nedko Ivanov for many useful discussions. This work has been supported by the DEG (Project Nr. Ri 615/6-1).

\section{References}

[1] W. Marshall, Proc. R. Soc. Lond. A 232, 48 (1955).

[2] E.H. Lieb, T.D. Schultz, D.C. Mattis, Ann. Phys. (N.Y.) 16, 407 (1961).

[3] K. Retzlaff, J. Richter, N.B. Ivanov, Z. Phys. B 93, 21 (1993).

[4] H. de Readt, A. Lagendijk, Phys. Rev. Lett. 46, 77 (1981).

[5] U. Schollwöck, Phys. Rev. B 58, 8194 (1998).

[6] C. Zeng, J.B. Parkinson, Phys. Rev. B 51, 11609 (1995).

[7] J. Richter, N.B. Ivanov, K. Retzlaff, Europhys. Lett. 25, 545 (1994).

[8] R.F. Bishop, D.J.J. Farnell, J.B. Parkinson, Phys. Rev. B 58, 6394 (1998).

[9] A. Voigt, J. Richter, N.B. Ivanov, Physica A 245, 269 (1997).

[10] F.D.M. Haldane, Phys. Rev. Lett. 60, 35 (1988). 